\title{
On a difference scheme of the second order of accuracy for elliptic-parabolic equations
}

\author{
Okan Gercek $^{1 *}$ and Allaberen Ashyralyev ${ }^{1,2}$
}

\section{"Correspondence:}

ogercek@fatih.edu.tr

1 Department of Mathematics, Fatih

University, Büyükçekmece, Istanbul,

Turkey

Full list of author information is

available at the end of the article

\begin{abstract}
The second order of accuracy difference scheme generated by Crank-Nicholson difference scheme for approximately solving multipoint nonlocal boundary value problem is considered. Well-posedness of this difference scheme in Hölder spaces is established. Furthermore, as applications, coercivity estimates in Hölder norms for approximate solutions of the multipoint nonlocal boundary value problems for mixed type equations are obtained. Moreover, the method is illustrated by numerical examples.
\end{abstract}

Keywords: difference scheme; elliptic-parabolic equation; well-posedness

\section{Introduction}

In recent years, more and more mathematicians have been studying nonlocal problems for ordinary differential equations and partial differential equations because of their existence in many applied problems included in applied sciences. Theory and numerical methods of solutions of the nonlocal boundary value problems for these partial differential equations were investigated by many researchers (see, e.g., [1-13] and the references therein). Several types of problems in fluid mechanics, other areas of physics, and mathematical biology led to partial differential equations of elliptic-parabolic type (see, [14-18]). The purpose of this paper is to study the second order of accuracy difference schemes of elliptic-parabolic problem with nonlocal boundary value problems.

In [19], we established the well-posedness of multipoint nonlocal boundary value problem

$$
\left\{\begin{array}{l}
-\frac{d^{2} u(t)}{d t^{2}}+A u(t)=g(t) \quad(0 \leq t \leq 1), \\
\frac{d u(t)}{d t}-A u(t)=f(t) \quad(-1 \leq t \leq 0), \\
u(1)=\sum_{i=1}^{J} \alpha_{i} u\left(\lambda_{i}\right)+\varphi, \quad-1 \leq \lambda_{1}<\cdots<\lambda_{J} \leq 0
\end{array}\right.
$$

in a Hilbert space $H$ with the self-adjoint positive definite operator $A$ under assumption

$$
\sum_{i=1}^{J}\left|\alpha_{i}\right| \leq 1
$$

The well-posedness of multipoint nonlocal boundary value problem (1) in Hölder spaces with a weight was established. In applications, coercivity inequalities for the solutions of nonlocal boundary value problems for elliptic-parabolic equations were obtained.

(c) 2012 Gercek and Ashyralyev; licensee Springer. This is an Open Access article distributed under the terms of the Creative Commons Attribution License (http://creativecommons.org/licenses/by/2.0), which permits unrestricted use, distribution, and reproduction in any medium, provided the original work is properly cited. 
In [20], we studied the well-posedness of the first order of accuracy difference scheme for the approximate solution of boundary value problem (1) under assumption (2).

In the present paper, we consider the second order of accuracy difference scheme generated by Crank-Nicholson difference scheme

$$
\left\{\begin{array}{l}
-\tau^{-2}\left(u_{k+1}-2 u_{k}+u_{k-1}\right)+A u_{k}=g_{k}, \\
g_{k}=g\left(t_{k}\right), \quad t_{k}=k \tau, 1 \leq k \leq N-1, N \tau=1, \\
\tau^{-1}\left(u_{k}-u_{k-1}\right)-\frac{1}{2}\left(A u_{k-1}+A u_{k}\right)=f_{k}, \\
f_{k}=f\left(t_{k-\frac{1}{2}}\right), \quad t_{k-\frac{1}{2}}=\left(k-\frac{1}{2}\right) \tau,-(N-1) \leq k \leq 0, \\
u_{2}-4 u_{1}+3 u_{0}=-3 u_{0}+4 u_{-1}-u_{-2}, \\
u_{N}=\sum_{k=1}^{J} \alpha_{i}\left(u_{\left[\frac{\lambda_{i}}{\tau}\right]}+\left(\lambda_{i}-\left[\frac{\lambda_{i}}{\tau}\right] \tau\right)\left(f_{\left[\frac{\lambda_{i}}{\tau}\right]}+A u_{\left[\frac{\lambda_{i}}{\tau}\right]}\right)\right)+\varphi
\end{array}\right.
$$

for the approximate solution of boundary value problem (1) under assumption (2).

The well-posedness of difference scheme (3) in Hölder spaces is established. In applications, the stability, almost coercivity stability, coercivity stability estimates for solutions of the second order of accuracy difference scheme for elliptic-parabolic equations are obtained. Furthermore, the theoretical statements for the solution of the first and second order of accuracy schemes for one-dimensional elliptic-parabolic differential equation are supported by the results of a numerical example.

\section{Main theorems}

Let us give some auxiliary lemmas we need below. Throughout the paper, $H$ is a Hilbert space and we denote $B=\frac{1}{2}\left(\tau A+\sqrt{A\left(4+\tau^{2} A\right)}\right)$, where $A$ is a self-adjoint positive definite operator. Then, it is clear that $B$ is a self-adjoint positive definite operator and $B \geq \delta^{\frac{1}{2}} I$, where $\delta>\delta_{0}>0$, and $R=(I+\tau B)^{-1}$ which is defined on the whole space $H$ is a bounded operator. The following operators

$$
P=\left(I-\frac{\tau A}{2}\right) G, \quad G=\left(I+\frac{\tau A}{2}\right)^{-1}, \quad R=(I+\tau B)^{-1}
$$

and

$$
\begin{aligned}
T_{\tau}= & \left(I+B^{-1} A\left(I+\tau A+\frac{\tau}{2} G^{-2}\right) K\left(I-R^{2 N-1}\right)+K\left(I-\frac{\tau^{2} A}{2}\right) G^{-2} R^{2 N-1}\right. \\
& \left.-K\left(I-\frac{\tau^{2} A}{2}\right) G^{-2}(2 I+\tau B) R^{N}\left[\sum_{i=1}^{n} \alpha_{i}\left(I+\left(\lambda_{i}-\left[\frac{\lambda_{i}}{\tau}\right] \tau\right) A\right) P^{-\left[\frac{\left.\lambda_{i}\right]}{\tau}\right]} u_{0}\right]\right)^{-1}
\end{aligned}
$$

exist and are bounded for a self-adjoint positive operator $A$. Here,

$$
B=\frac{1}{2}\left(\tau A+\sqrt{A\left(4+\tau^{2} A\right)}\right), \quad K=\left(I+2 \tau A+\frac{5}{4}(\tau A)^{2}\right)^{-1},
$$

and $I$ is the identity operator. 
Lemma 2.1 For any $g_{k}, 1 \leq k \leq N-1$ and $f_{k},-N+1 \leq k \leq 0$, the solution of problem (3) exists and the following formulas hold:

$$
\begin{aligned}
& u_{k}=\left(I-R^{2 N}\right)^{-1}\left\{\left[R^{k}-R^{2 N-k}\right] u_{0}+\left[R^{N-k}-R^{N+k}\right]\right. \\
& \times\left[\sum _ { i = 1 } ^ { n } \alpha _ { i } \left[\left(I+\left(\lambda_{i}-\left[\frac{\lambda_{i}}{\tau}\right] \tau\right) A\right)\right.\right.
\end{aligned}
$$

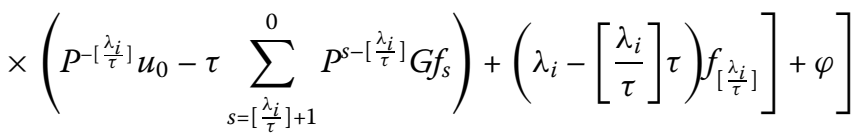

$$
\begin{aligned}
& \left.-\left[R^{N-k}-R^{N+k}\right](I+\tau B)(2 I+\tau B)^{-1} B^{-1} \sum_{s=1}^{N-1}\left[R^{N-s}-R^{N+s}\right] g_{s} \tau\right\} \\
& +(I+\tau B)(2 I+\tau B)^{-1} B^{-1} \sum_{s=1}^{N-1}\left[R^{|k-s|}-R^{k+s}\right] g_{s} \tau, \quad 1 \leq k \leq N, \\
& u_{k}=P^{-k} u_{0}-\tau \sum_{s=k+1}^{0} P^{s-k-1} G f_{s}, \quad-N \leq k \leq-1, \\
& u_{0}=\frac{1}{2} T_{\tau} K G^{-2} \times\left\{( 2 I - \tau ^ { 2 } A ) \left\{( 2 + \tau B ) R ^ { N } \left[\sum_{i=1}^{n} \alpha_{i}\left(I+\left(\lambda_{i}-\left[\frac{\lambda_{i}}{\tau}\right] \tau\right) A\right)\right.\right.\right. \\
& \left.\times\left(-\tau \sum_{s=\left[\frac{\lambda_{i}}{\tau}\right]+1}^{0} P^{s-\left[\frac{\lambda_{i}}{\tau}\right]} G f_{s}\right)+\left(\lambda_{i}-\left[\frac{\lambda_{i}}{\tau}\right] \tau\right) f_{\left[\frac{\lambda_{i}}{\tau}\right]}+\varphi\right] \\
& \left.-R^{N-1} B^{-1} \sum_{s=1}^{N-1}\left[R^{N-s}-R^{N+s}\right] g_{s} \tau+\left(I-R^{2 N}\right) B^{-1} \sum_{s=1}^{N-1} R^{s-1} g_{s} \tau\right\} \\
& \left.+\left(I-R^{2 N}\right)(I+\tau B)\left(\tau B^{-1} g_{1}-4 G B^{-1} f_{0}+P G B^{-1} f_{0}+G B^{-1} f_{-1}\right)\right\}, \\
& T_{\tau}=\left(I+B^{-1} A\left(I+\tau A+\frac{\tau}{2} G^{-2}\right) K\left(I-R^{2 N-1}\right)+K\left(I-\frac{\tau^{2} A}{2}\right) G^{-2} R^{2 N-1}\right. \\
& \left.-K\left(I-\frac{\tau^{2} A}{2}\right) G^{-2}(2 I+\tau B) R^{N}\left[\sum_{i=1}^{n} \alpha_{i}\left(I+\left(\lambda_{i}-\left[\frac{\lambda_{i}}{\tau}\right] \tau\right) A\right) P^{-\left[\frac{\lambda_{i}}{\tau}\right]} u_{0}\right]\right)^{-1} .
\end{aligned}
$$

Proof Clearly, the solution formula of the problem

$$
\tau^{-1}\left(u_{k}-u_{k-1}\right)-\frac{1}{2}\left(A u_{k-1}+A u_{k}\right)=f_{k}, \quad-(N-1) \leq k \leq 0, u_{0}=\gamma
$$

is [22]:

$$
u_{k}=P^{-k} \gamma-\tau \sum_{s=k+1}^{0} P^{s-k-1} G f_{s}, \quad-N \leq k \leq-1
$$

for any $\left\{f_{k}\right\}_{k=-N}^{-1}$ and $\gamma$. Equation (9) and the fact that $u_{0}=\gamma$ yield Equation (6). 
The solution of the problem

$$
\left\{\begin{array}{l}
-\tau^{-2}\left(u_{k+1}-2 u_{k}+u_{k-1}\right)+A u_{k}=g_{k}, \\
g_{k}=g\left(t_{k}\right), \quad t_{k}=k \tau, 1 \leq k \leq N-1, \quad u_{0}=\gamma, \quad u_{N}=\psi
\end{array}\right.
$$

satisfies the following formula [21]:

$$
\begin{aligned}
u_{k}= & \left(I-R^{2 N}\right)^{-1}\left\{\left[R^{k}-R^{2 N-k}\right] \gamma+\left[R^{N-k}-R^{N+k}\right] \psi\right. \\
& \left.-\left[R^{N-k}-R^{N+k}\right](I+\tau B)(2 I+\tau B)^{-1} B^{-1} \sum_{s=1}^{N-1}\left[R^{N-s}-R^{N+s}\right] g_{s} \tau\right\} \\
& +(I+\tau B)(2 I+\tau B)^{-1} B^{-1} \sum_{s=1}^{N-1}\left[R^{|k-s|}-R^{k+s}\right] g_{s} \tau, \quad 1 \leq k \leq N .
\end{aligned}
$$

Equation (5) follows from Equations (9) and (11), initial condition $u_{0}=\gamma$, and

$$
\psi=\sum_{k=1}^{J} \alpha_{i}\left(u_{\left[\frac{\lambda_{i}}{\tau}\right]}+\left(\lambda_{i}-\left[\frac{\lambda_{i}}{\tau}\right] \tau\right)\left(f_{\left[\frac{\lambda_{i}}{\tau}\right]}+A u_{\left[\frac{\lambda_{i}}{\tau}\right]}\right)\right)+\varphi
$$

Finally, let us obtain formula (7). Combining (5), (6), and the condition

$$
u_{2}-4 u_{1}+3 u_{0}=-3 u_{0}+4 u_{-1}-u_{-2},
$$

we get

$$
\begin{aligned}
(2 I- & \left.\tau^{2} A\right)\left\{( I - R ^ { 2 N } ) ^ { - 1 } \left\{\left[R-R^{2 N-1}\right] u_{0}+\left[R^{N-1}-R^{N+1}\right]\left[\sum_{i=1}^{n} \alpha_{i}\left(I+\left(\lambda_{i}-\left[\frac{\lambda_{i}}{\tau}\right] \tau\right) A\right)\right.\right.\right. \\
& \left.\times\left(P^{-\left[\frac{\lambda_{i}}{\tau}\right]} u_{0}-\tau \sum_{s=\left[\frac{\lambda_{i}}{\tau}\right]+1}^{0} P^{s-\left[\frac{\lambda_{i}}{\tau}\right]} f_{s}\right)+\sum_{i=1}^{n} \alpha_{i}\left(\lambda_{i}-\left[\frac{\lambda_{i}}{\tau}\right] \tau\right) f_{\left[\frac{\lambda_{i}}{\tau}\right]}+\varphi\right] \\
& \left.-\left[R^{N-1}-R^{N+1}\right](I+\tau B)(2 I+\tau B)^{-1} B^{-1} \sum_{s=1}^{N-1}\left[R^{N-s}-R^{N+s}\right] g_{s} \tau\right\} \\
& \left.+(I+\tau B)(2 I+\tau B)^{-1} B^{-1} \sum_{s=1}^{N-1}\left[R^{s-1}-R^{1+s}\right] g_{s} \tau\right\} \\
= & -\tau^{2} g_{1}+G^{2}\left(2 I+4 \tau A+\frac{5}{2}(\tau A)^{2}\right) u_{0}+4 G \tau f_{0}-P G \tau f_{0}-G \tau f_{-1} .
\end{aligned}
$$

From Equation (4), it follows that

$$
\begin{aligned}
u_{0}= & \frac{1}{2} T_{\tau} K G^{-2} \times\left\{\left(2 I-\tau^{2} A\right)\right\}(2+\tau B) R^{N} \\
& \times\left[\sum_{i=1}^{n} \alpha_{i}\left(I+\left(\lambda_{i}-\left[\frac{\lambda_{i}}{\tau}\right] \tau\right) A\right)\left(-\tau \sum_{s=\left[\frac{\lambda_{i}}{\tau}\right]+1}^{0} P^{s-\left[\frac{\lambda_{i}}{\tau}\right]} G f_{s}\right)\right.
\end{aligned}
$$




$$
\begin{aligned}
& \left.+\left(\lambda_{i}-\left[\frac{\lambda_{i}}{\tau}\right] \tau\right) f_{\left[\frac{\lambda_{i}}{\tau}\right]}+\varphi\right] \\
& \left.-R^{N-1} B^{-1} \sum_{s=1}^{N-1}\left[R^{N-s}-R^{N+s}\right] g_{s} \tau+\left(I-R^{2 N}\right) B^{-1} \sum_{s=1}^{N-1} R^{s-1} g_{s} \tau\right\} \\
& \left.+\left(I-R^{2 N}\right)(I+\tau B)\left(\tau B^{-1} g_{1}-4 G B^{-1} f_{0}+P G B^{-1} f_{0}+G B^{-1} f_{-1}\right)\right\} .
\end{aligned}
$$

This finishes the proof of Lemma 2.1.

Here, we study well-posedness of problem (3). First, we give some necessary estimates for $P^{k}, R^{k}$ and $T_{\tau}$.

Lemma 2.2 For a self-adjoint positive operator A, the following estimates are satisfied [21, 22, 24]:

$$
\left\{\begin{array}{l}
\left\|P^{k}\right\|_{H \rightarrow H} \leq 1, \quad k \tau\left\|A P^{k} G^{2}\right\|_{H \rightarrow H} \leq M(\delta), \quad k \tau\left\|B R^{k}\right\|_{H \rightarrow H} \leq M(\delta), \\
\left\|R^{k}\right\|_{H \rightarrow H} \leq M(\delta)(1+\delta \tau)^{-k}, \quad\left\|\left(I-R^{2 N}\right)^{-1}\right\|_{H \rightarrow H} \leq M(\delta), \quad\|G\|_{H \rightarrow H} \leq 1, \\
\left\|P^{k}-e^{-k \tau A}\right\|_{H \rightarrow H} \leq \frac{M(\delta) \tau}{k \tau}, \quad\left\|R^{k}-e^{-k \tau A^{\frac{1}{2}}}\right\|_{H \rightarrow H} \leq \frac{M(\delta) \tau}{k \tau}, \quad k \geq 1, \delta>0,
\end{array}\right.
$$

where $M(\delta)$ is independent of $\tau$.

From these estimates, it follows that

$$
\begin{aligned}
\|(I+ & B^{-1} A\left(I+\tau A+\frac{\tau}{2} G^{-2}\right) K\left(I-R^{2 N-1}\right) \\
& +K\left(I-\frac{\tau^{2} A}{2}\right) G^{-2} R^{2 N-1}-K\left(I-\frac{\tau^{2} A}{2}\right) G^{-2}(2 I+\tau B) R^{N} \\
& \left.\times\left[\sum_{i=1}^{n} \alpha_{i}\left(I+\left(\lambda_{i}-\left[\frac{\lambda_{i}}{\tau}\right] \tau\right) A\right) P^{-\left[\frac{\left.\lambda_{i}\right]}{\tau}\right]} u_{0}\right]\right)^{-1} \|_{H \rightarrow H} \\
\leq & M(\delta) .
\end{aligned}
$$

Now, we study well-posedness of problem (3). Let $F_{\tau}(H)=F\left([a, b]_{\tau}, H\right)$ be the linear space of mesh functions $\varphi^{\tau}=\left\{\varphi_{k}\right\}_{\widetilde{N}} \widetilde{\widetilde{N}}$ defined on $[a, b]_{\tau}=\left\{t_{k}=k h, \widetilde{N} \leq k \leq \widetilde{\widetilde{N}}, \widetilde{N} \tau=\right.$ $a, \widetilde{\widetilde{N}} \tau=b\}$ with values in the Hilbert space $H$. Next, on $F_{\tau}(H)$ we denote $C\left([a, b]_{\tau}, H\right)$, $C_{0,1}^{\alpha}\left([-1,1]_{\tau}, H\right), C_{0,1}^{\alpha}\left([-1,0]_{\tau}, H\right), C_{0}^{\alpha}\left([0,1]_{\tau}, H\right), \widetilde{C}_{0,1}^{\alpha}\left([-1,1]_{\tau}, H\right)$, and $\widetilde{C}_{0}^{\alpha}\left([-1,0]_{\tau}, H\right), 0<$ $\alpha<1$ Banach spaces with the norms

$$
\begin{aligned}
\left\|\varphi^{\tau}\right\|_{C\left([a, b]_{\tau}, H\right)}= & \max _{N_{a} \leq k \leq N_{b}}\left\|\varphi_{k}\right\|_{H}, \\
\left\|\varphi^{\tau}\right\|_{C_{0,1}^{\alpha}\left([-1,1]_{\tau}, H\right)}= & \left\|\varphi^{\tau}\right\|_{C\left([-1,1]_{\tau}, H\right)}+\sup _{-N \leq k<k+r \leq 0}\left\|\varphi_{k+r}-\varphi_{k}\right\|_{E}(-k)^{\alpha} r^{-\alpha} \\
& +\sup _{1 \leq k<k+r \leq N-1}\left\|\varphi_{k+r}-\varphi_{k}\right\|_{E}((k+r) \tau)^{\alpha}(N-k)^{\alpha} r^{-\alpha},
\end{aligned}
$$




$$
\begin{aligned}
\left\|\varphi^{\tau}\right\|_{C_{0}^{\alpha}\left([-1,0]_{\tau}, H\right)}= & \left\|\varphi^{\tau}\right\|_{C\left([-1,0]_{\tau}, H\right)}+\sup _{-N \leq k<k+r \leq 0}\left\|\varphi_{k+r}-\varphi_{k}\right\|_{E}(-k)^{\alpha} r^{-\alpha}, \\
\left\|\varphi^{\tau}\right\|_{C_{0,1}^{\alpha}\left([0,1]_{\tau}, H\right)}= & \left\|\varphi^{\tau}\right\|_{C\left([0,1]_{\tau}, H\right)} \\
& +\sup _{1 \leq k<k+r \leq N-1}\left\|\varphi_{k+r}-\varphi_{k}\right\|_{E}((k+r) \tau)^{\alpha}(N-k)^{\alpha} r^{-\alpha}, \\
\left\|\varphi^{\tau}\right\|_{\tilde{C}_{0,1}^{\alpha}\left[\left([-1,1]_{\tau}, H\right)\right.}= & \left\|\varphi^{\tau}\right\|_{C\left([-1,1]_{\tau}, H\right)}+\sup _{-N \leq k<k+2 r \leq 0}\left\|\varphi_{k+2 r}-\varphi_{k}\right\|_{E}(-k)^{\alpha}(2 r)^{-\alpha} \\
& +\sup _{1 \leq k<k+r \leq N-1}\left\|\varphi_{k+r}-\varphi_{k}\right\|_{E}((k+r) \tau)^{\alpha}(N-k)^{\alpha} r^{-\alpha}, \\
\left\|\varphi^{\tau}\right\|_{\widetilde{C}_{0}^{\alpha}\left([-1,0]_{\tau}, H\right)}= & \left\|\varphi^{\tau}\right\|_{C\left([-1,0]_{\tau}, H\right)}+\sup _{-N \leq k<k+2 r \leq 0}\left\|\varphi_{k+2 r}-\varphi_{k}\right\|_{E}(-k)^{\alpha}(2 r)^{-\alpha} .
\end{aligned}
$$

Theorem 2.1 Nonlocal boundary value problem (3) is stable in $C\left([-1,1]_{\tau}, H\right)$ norm.

Proof By [21], we have

$$
\left\|\left\{u_{k}\right\}_{1}^{N-1}\right\|_{C\left([0,1]_{\tau}, H\right)} \leq M\left[\left\|g^{\tau}\right\|_{C\left([0,1]_{\tau}, H\right)}+\left\|u_{0}\right\|_{H}+\left\|u_{N}\right\|_{H}\right]
$$

for the solution of boundary value problem (10).

By [22], we have

$$
\left\|\left\{u_{k}\right\}_{-N}^{0}\right\|_{C\left([-1,0]_{\tau}, H\right)} \leq M\left[\left\|f^{\tau}\right\|_{C\left([-1,0]_{\tau}, H\right)}+\left\|u_{0}\right\|_{H}\right]
$$

for the solution of inverse Cauchy difference problem (8).

Then, the proof of Theorem 2.1 is based on stability inequalities (14), (15), and on estimates

$$
\begin{aligned}
& \left\|u_{0}\right\|_{H} \leq M(\delta)\left[\left\|f^{\tau}\right\|_{C\left([-1,0]_{\tau}, H\right)}+\left\|g^{\tau}\right\|_{C\left([0,1]_{\tau}, H\right)}+\|\varphi\|_{H}\right], \\
& \left\|u_{N}\right\|_{H} \leq M(\delta)\left[\left\|f^{\tau}\right\|_{C\left([-1,0]_{\tau}, H\right)}+\left\|g^{\tau}\right\|_{C\left([0,1]_{\tau}, H\right)}+\|\varphi\|_{H}\right]
\end{aligned}
$$

for the solution of boundary value problem (3). Estimates (16) and (17) follow from formula (7) and estimates (12), (13). Theorem 2.1 is proved.

Theorem 2.2 Assume that $\varphi \in D(A)$ and $f_{0}, f_{-1}, g_{1} \in D(I+\tau B)$. Then, for the solution of difference problem (3), we have the following almost coercivity inequality:

$$
\begin{aligned}
& \left\|\left\{\tau^{-2}\left(u_{k+1}-2 u_{k}+u_{k-1}\right)\right\}_{1}^{N-1}\right\|_{C\left([0,1]_{\tau}, H\right)} \\
& \quad+\left\|\left\{\tau^{-1}\left(u_{k}-u_{k-1}\right)\right\}_{-N+1}^{0}\right\|_{C\left([-1,0]_{\tau}, H\right)} \\
& \quad+\left\|\left\{A u_{k}\right\}_{1}^{N-1}\right\|_{C\left([0,1]_{\tau}, H\right)}+\left\|\left\{\frac{1}{2}\left(A u_{k}+A u_{k-1}\right)\right\}_{-N+1}^{0}\right\|_{C\left([-1,0]_{\tau}, H\right)} \\
& \leq M(\delta)\left[\min \left\{\ln \frac{1}{\tau}, 1+\left|\ln \|A\|_{H \rightarrow H}\right|\right\}\left[\left\|f^{\tau}\right\|_{C\left([-1,0]_{\tau}, H\right)}+\left\|g^{\tau}\right\|_{C\left([0,1]_{\tau}, H\right)}\right]\right. \\
& \left.\quad+\|A \varphi\|_{H}+\left\|(I+\tau B) f_{0}\right\|_{H}+\left\|(I+\tau B) g_{1}\right\|_{H}+\left\|(I+\tau B) f_{-1}\right\|_{H}\right],
\end{aligned}
$$

where $M(\delta)$ is independent not only off $f^{\tau}, g^{\tau}, \varphi$ but also of $\tau$. 
Proof By [24], we have

$$
\begin{aligned}
& \left\|\left\{\tau^{-1}\left(u_{k}-u_{k-1}\right)\right\}_{-N+1}^{0}\right\|_{C\left([-1,0]_{\tau}, H\right)} \\
& +\left\|\left\{\frac{1}{2}\left(A u_{k}+A u_{k-1}\right)\right\}_{-N+1}^{0}\right\| \|_{C\left([-1,0]_{\tau}, H\right)} \\
& \leq M(\delta)\left[\min \left\{\ln \frac{1}{\tau}, 1+\left|\ln \|A\|_{H \rightarrow H}\right|\right\}\left\|f^{\tau}\right\|_{C\left([-1,0]_{\tau}, H\right)}+\left\|A u_{0}\right\|_{H}\right]
\end{aligned}
$$

for the solution of inverse Cauchy difference problem (8).

By [21], we have

$$
\begin{aligned}
& \left\|\left\{\tau^{-2}\left(u_{k+1}-2 u_{k}+u_{k-1}\right)\right\}_{1}^{N-1}\right\|_{C\left([0,1]_{\tau}, H\right)}+\left\|\left\{A u_{k}\right\}_{1}^{N-1}\right\|_{C\left([0,1]_{\tau}, H\right)} \\
& \quad \leq M(\delta)\left[\min \left\{\ln \frac{1}{\tau}, 1+\left|\ln \|A\|_{H \rightarrow H}\right|\right\}\left\|g^{\tau}\right\|_{C\left([0,1]_{\tau}, H\right)}+\left\|A u_{0}\right\|_{H}+\left\|A u_{N}\right\|_{H}\right]
\end{aligned}
$$

for the solution of boundary value problem (10). Then, the proof of Theorem 2.2 is based on almost coercivity inequalities (18), (19), and on the estimates

$$
\begin{aligned}
& \left\|A u_{0}\right\|_{H} \leq M(\delta)\left[\|A \varphi\|_{H}+\left\|(I+\tau B) f_{0}\right\|_{H}\right. \\
& \left.+\min \left\{\ln \frac{1}{\tau}, 1+\left|\ln \|A\|_{H \rightarrow H}\right|\right\}\left[\left\|f^{\tau}\right\|_{C\left([-1,0]_{\tau}, H\right)}+\left\|g^{\tau}\right\|_{C\left([0,1]_{\tau}, H\right)}\right]\right], \\
& \left\|A u_{N}\right\|_{H} \leq M(\delta)\left[\left[\|A \varphi\|_{H}+\left\|(I+\tau B) f_{0}\right\|_{H}\right]\right. \\
& \left.+\min \left\{\ln \frac{1}{\tau}, 1+\left|\ln \|A\|_{H \rightarrow H}\right|\right\}\left[\left\|f^{\tau}\right\|_{C\left([-1,0]_{\tau}, H\right)}+\left\|g^{\tau}\right\|_{C\left([0,1]_{\tau}, H\right)}\right]\right]
\end{aligned}
$$

for the solution of boundary value problem (3). The proof of these estimates follows the scheme of papers [21, 24] and relies on both formula (7) and estimates (12), (13). This finalizes the proof of Theorem 2.2 .

Theorem 2.3 Let the assumptions of Theorem 2.2 be satisfied. Then, boundary value problem (3) is well-posed in Hölder spaces $C_{0,1}^{\alpha}\left([-1,1]_{\tau}, H\right), \widetilde{C}_{0,1}^{\alpha}\left([-1,1]_{\tau}, H\right)$ and the following coercivity inequalities hold:

$$
\begin{aligned}
& \left\|\left\{\tau^{-2}\left(u_{k+1}-2 u_{k}+u_{k-1}\right)\right\}_{1}^{N-1}\right\|_{C_{0,1}^{\alpha}\left([0,1]_{\tau}, H\right)} \\
& \quad+\left\|\left\{\tau^{-1}\left(u_{k}-u_{k-1}\right)\right\}_{-N+1}^{0}\right\|_{\widetilde{C}_{0}^{\alpha}\left([-1,0]_{\tau}, H\right)} \\
& \quad+\left\|\left\{A u_{k}\right\}_{1}^{N-1}\right\|_{C_{0,1}^{\alpha}\left([0,1]_{\tau}, H\right)}+\left\|\left\{\frac{1}{2}\left(A u_{k}+A u_{k-1}\right)\right\}_{-N+1}\right\|_{\widetilde{C}_{0}^{\alpha}\left([-1,0]_{\tau}, H\right)} \\
& \leq M(\delta)\left[\frac{1}{\alpha(1-\alpha)}\left[\left\|f^{\tau}\right\|_{C_{0}^{\alpha}\left([-1,0]_{\tau}, H\right)}+\left\|g^{\tau}\right\|_{C_{0,1}^{\alpha}\left([0,1]_{\tau}, H\right)}\right]+\|A \varphi\|_{H}\right. \\
& \left.\quad+\left\|(I+\tau B) f_{0}\right\|_{H}+\left\|(I+\tau B) g_{1}\right\|_{H}+\left\|(I+\tau B) f_{-1}\right\|_{H}\right] \\
& \left\|\left\{\tau^{-2}\left(u_{k+1}-2 u_{k}+u_{k-1}\right)\right\}_{1}^{N-1}\right\|_{C_{0,1}^{\alpha}\left([0,1]_{\tau}, H\right)}
\end{aligned}
$$




$$
\begin{aligned}
& +\left\|\left\{\tau^{-1}\left(u_{k}-u_{k-1}\right)\right\}_{-N+1}^{0}\right\|_{\widetilde{C}_{0}^{\alpha}\left([-1,0]_{\tau}, H\right)} \\
& +\left\|\left\{A u_{k}\right\}_{1}^{N-1}\right\|_{C_{0,1}^{\alpha}\left([0,1]_{\tau}, H\right)}+\left\|\left\{\frac{1}{2}\left(A u_{k}+A u_{k-1}\right)\right\}_{-N+1}^{0}\right\| \\
\leq & M(\delta)\left[\frac { 1 } { \alpha ( 1 - \alpha ) } \left[\left\|f^{\tau}\right\|_{\widetilde{C}_{0}^{\alpha}\left([-1,0]_{\tau}, H\right)}\left([-1,0]_{\tau}, H\right)\right.\right. \\
& \left.+\left\|(I+\tau B) f_{0}\right\|_{H}+\left\|(I+\tau B) g_{1}\right\|_{C_{0,1}^{\alpha}\left([0,1]_{\tau}, H\right)}+\left\|(I+\tau B) f_{-1}\right\|_{H}\right],\|A \varphi\|_{H}
\end{aligned}
$$

where $M$ is independent of $f^{\tau}, g^{\tau}, \varphi, \tau$ and $\alpha$.

Proof By [24],

$$
\begin{aligned}
& \left\|\left\{\tau^{-1}\left(u_{k}-u_{k-1}\right)\right\}_{-N+1}^{0}\right\|_{\widetilde{C}_{0}^{\alpha}\left([-1,0]_{\tau}, H\right)} \\
& +\left\|\left\{\frac{1}{2}\left(A u_{k}+A u_{k-1}\right)\right\}_{-N+1}^{0}\right\|_{\widetilde{C}_{0}^{\alpha}\left([-1,0]_{\tau}, H\right)} \\
& \leq M(\delta)\left[\frac{1}{\alpha(1-\alpha)}\left\|f^{\tau}\right\|_{C_{0}^{\alpha}\left([-1,0]_{\tau}, H\right)}+\left\|A u_{0}\right\|_{H}\right], \\
& \left\|\left\{\tau^{-1}\left(u_{k}-u_{k-1}\right)\right\}_{-N+1}^{0}\right\| \|_{\widetilde{C}_{0}^{\alpha}\left([-1,0]_{\tau}, H\right)} \\
& +\left\|\left\{\frac{1}{2}\left(A u_{k}+A u_{k-1}\right)\right\}_{-N+1}^{0}\right\| \|_{\widetilde{C}_{0}^{\alpha}\left([-1,0]_{\tau}, H\right)} \\
& \leq M(\delta)\left[\frac{1}{\alpha(1-\alpha)}\left\|f^{\tau}\right\|_{\widetilde{C}_{0}^{\alpha}\left([-1,0]_{\tau}, H\right)}+\left\|A u_{0}\right\|_{H}\right]
\end{aligned}
$$

for the solution of inverse Cauchy difference problem (8).

By [21], we have

$$
\begin{aligned}
& \left\|\left\{\tau^{-2}\left(u_{k+1}-2 u_{k}+u_{k-1}\right)\right\}_{1}^{N-1}\right\|_{C_{0,1}^{\alpha}\left([0,1]_{\tau}, H\right)}+\left\|\left\{A u_{k}\right\}_{1}^{N-1}\right\|_{C_{0,1}^{\alpha}\left([0,1]_{\tau}, H\right)} \\
& \quad \leq M(\delta)\left[\frac{1}{\alpha(1-\alpha)}\left\|g^{\tau}\right\|_{C_{0,1}^{\alpha}\left([0,1]_{\tau}, H\right)}+\left\|A u_{0}\right\|_{H}+\left\|A u_{N}\right\|_{H}\right]
\end{aligned}
$$

for the solution of boundary value problem (10). Then, the proof of Theorem 2.3 is based on coercivity inequalities (20)-(22) and estimates

$$
\begin{aligned}
\left\|A u_{0}\right\|_{H} \leq & M(\delta)\left[\frac{1}{\alpha(1-\alpha)}\left[\left\|f^{\tau}\right\|_{\widetilde{C}_{0}^{\alpha}\left([-1,0]_{\tau}, H\right)}+\left\|g^{\tau}\right\|_{C_{0,1}^{\alpha}\left([0,1]_{\tau}, H\right)}\right]\right. \\
& \left.+\|A \varphi\|_{H}+\left\|(I+\tau B) f_{0}\right\|_{H}+\left\|(I+\tau B) g_{1}\right\|_{H}+\left\|(I+\tau B) f_{-1}\right\|_{H}\right], \\
\left\|A u_{N}\right\|_{H} \leq & M(\delta)\left[\frac{1}{\alpha(1-\alpha)}\left[\left\|f^{\tau}\right\|_{\widetilde{C}_{0}^{\alpha}\left([-1,0]_{\tau}, H\right)}+\left\|g^{\tau}\right\|_{C_{0,1}^{\alpha}\left([0,1]_{\tau}, H\right)}\right]\right. \\
& \left.+\|A \varphi\|_{H}+\left\|(I+\tau B) f_{0}\right\|_{H}+\left\|(I+\tau B) g_{1}\right\|_{H}+\left\|(I+\tau B) f_{-1}\right\|_{H}\right]
\end{aligned}
$$


for the solution of difference scheme (3). The proof of these estimates follows the scheme of the papers [21, 24] and relies on both formula (7) and estimates (12), (13). This is the end of the proof of Theorem 2.3.

\section{Application}

Now, the application of the abstract result is considered. In $[-1,1] \times \Omega$, let us consider the boundary value problem for multi-dimensional elliptic-parabolic equation

$$
\left\{\begin{array}{l}
-u_{t t}-\sum_{r=1}^{n}\left(a_{r}(x) u_{x_{r}}\right)_{x_{r}}=g(t, x), \quad 0<t<1, x \in \Omega, \\
u_{t}+\sum_{r=1}^{n}\left(a_{r}(x) u_{x_{r}}\right)_{x_{r}}=f(t, x), \quad-1<t<0, x \in \Omega, \\
u(t, x)=0, \quad x \in S,-1 \leq t \leq 1 ; \\
u(1, x)=\sum_{i=1}^{J} \alpha_{i} u\left(\lambda_{i}, x\right)+\varphi(x), \quad \sum_{i=1}^{J}\left|\alpha_{i}\right| \leq 1, \\
-1 \leq \lambda_{1}<\lambda_{2}<\cdots<\lambda_{i}<\cdots<\lambda_{J} \leq 0, \\
u(0+, x)=u(0-, x), \quad u_{t}(0+, x)=u_{t}(0-, x), \quad x \in \bar{\Omega},
\end{array}\right.
$$

where $a_{r}(x)(x \in \Omega), \varphi(x)(\varphi(x)=0, x \in S), g(t, x)(t \in(0,1), x \in \bar{\Omega})$, and $f(t, x)(t \in(-1,0)$, $x \in \bar{\Omega})$ are given smooth functions. Here, $\Omega$ is the unit open cube in the $n$-dimensional Euclidean space $\mathbb{R}^{n}\left(0<x_{k}<1,1 \leq k \leq n\right)$ with boundary $S, \bar{\Omega}=\Omega \cup S$, and $a_{r}(x) \geq a>0$.

The discretization of problem (23) is carried out in two steps. In the first step, the grid sets

$$
\begin{aligned}
\widetilde{\Omega}_{h}= & \left\{x=x_{m}=\left(h_{1} m_{1}, \ldots, h_{h} m_{n}\right), m=\left(m_{1}, \ldots, m_{h}\right),\right. \\
& \left.0 \leq m_{r} \leq N_{r}, h_{r} N_{r}=1, r=1, \ldots, n\right\}, \\
\Omega_{h}= & \widetilde{\Omega}_{h} \cap \Omega, S_{h}=\widetilde{\Omega}_{h} \cap S
\end{aligned}
$$

are defined. To the differential operator $A$ generated by problem (23), we assign the difference operator $A_{h}^{x}$ by formula

$$
A_{h}^{x} u^{h}=-\sum_{r=1}^{n}\left(a_{r}(x) u_{\bar{x}_{r}}^{h}\right)_{x_{r}, m_{r}}
$$

acting in the space of grid functions $u^{h}(x)$, satisfying the conditions $u^{h}(x)=0$ for all $x \in S_{h}$. With the help of $A_{h}^{x}$, we arrive at the nonlocal boundary value problem

$$
\left\{\begin{array}{l}
-\frac{d^{2} u^{h}(t, x)}{d t^{2}}+A_{h}^{x} u^{h}(t, x)=g^{h}(t, x), \quad 0<t<1, x \in \Omega_{h}, \\
\frac{d u^{h}(t, x)}{d t}-A_{h}^{x} u^{h}(t, x)=f^{h}(t, x), \quad-1<t<0, x \in \Omega_{h}, \\
u^{h}(1, x)=u^{h}(-1, x)+\varphi^{h}(x), \quad x \in \widetilde{\Omega}_{h}, \\
u^{h}(0+, x)=u^{h}(0-, x), \quad \frac{d u^{h}(0+, x)}{d t}=\frac{d u^{h}(0-, x)}{d t}, \quad x \in \widetilde{\Omega}_{h}
\end{array}\right.
$$

for an infinite system of ordinary differential equations (see [21]). 
Secondly, problem (25) is replaced by difference scheme (3), so that the following second order of accuracy difference scheme

$$
\left\{\begin{array}{l}
-\frac{u_{k+1}^{h}(x)-2 u_{k}^{h}(x)+u_{k-1}^{h}(x)}{\tau^{2}}+A_{h}^{x} u_{k}^{h}(x)=g_{k}^{h}(x), \\
g_{k}^{h}(x)=g^{h}\left(t_{k}, x\right), \quad t_{k}=k \tau, 1 \leq k \leq N-1, N \tau=1, x \in \Omega_{h}, \\
\frac{u_{k}^{h}(x)-u_{k-1}^{h}(x)}{\tau}-\frac{A_{h}^{x}}{2}\left(u_{k}^{h}(x)+u_{k-1}^{h}(x)\right)=f_{k}^{h}(x), \\
f_{k}^{h}(x)=f^{h}\left(t_{k-\frac{1}{2}}, x\right), \quad t_{k-\frac{1}{2}}=\left(k-\frac{1}{2}\right) \tau,-N+1 \leq k \leq 0, x \in \Omega_{h}, \\
-u_{2}^{h}(x)+4 u_{1}^{h}(x)-3 u_{0}^{h}(x)=3 u_{0}^{h}(x)-4 u_{-1}^{h}(x)+u_{-2}^{h}(x), \quad x \in \widetilde{\Omega}_{h}, \\
\left.\left.u_{N}^{h}(x)=\sum_{k=1}^{J} \alpha_{i}\left(u_{\left[\frac{\lambda_{i}}{\tau}\right]}^{h}(x)+\left(\lambda_{k}-\left[\frac{\lambda_{i}}{\tau}\right] \tau\right)\right]_{\left[\frac{\lambda_{i}}{\tau}\right]}^{h}+A_{h}^{x} u_{\left[\frac{\lambda_{i}}{\tau}\right]}^{h}(x)\right)\right)+\varphi^{h}(x), \quad x \in \widetilde{\Omega}_{h} .
\end{array}\right.
$$

is obtained (see [21], [22]).

To formulate the results, we introduce the spaces $L_{2 h}=L_{2}\left(\bar{\Omega}_{h}\right), W_{2 h}^{1}=W_{2}^{1}\left(\bar{\Omega}_{h}\right)$, and $W_{2 h}^{2}=W_{2}^{2}\left(\bar{\Omega}_{h}\right)$ of the grid functions $\varphi^{h}(x)=\left\{\varphi\left(h_{1} m_{1}, \ldots, h_{n} m_{h}\right)\right\}$ defined on $\bar{\Omega}_{h}$, equipped with the norms

$$
\begin{aligned}
& \left\|\varphi^{h}\right\|_{L_{2 h}}=\left(\sum_{x \in \bar{\Omega}_{h}}\left|\varphi^{h}(x)\right|^{2} h_{1} \cdots h_{n}\right)^{1 / 2}, \\
& \left\|\varphi^{h}\right\|_{W_{2 h}^{1}}=\left\|\varphi^{h}\right\|_{L_{2 h}}+\left(\sum_{x \in \bar{\Omega}_{h}} \sum_{r=1}^{n}\left|\left(\varphi^{h}\right)_{x_{r}}\right|^{2} h_{1} \cdots h_{n}\right)^{1 / 2},
\end{aligned}
$$

and

$$
\begin{aligned}
\left\|\varphi^{h}\right\|_{W_{2 h}^{2}}= & \left\|\varphi^{h}\right\|_{L_{2 h}}+\left(\sum_{x \in \bar{\Omega}_{h}} \sum_{r=1}^{n}\left|\left(\varphi^{h}\right)_{x_{r}}\right|^{2} h_{1} \cdots h_{n}\right)^{1 / 2} \\
& +\left(\sum_{x \in \bar{\Omega}_{h}} \sum_{r=1}^{n}\left|\left(\varphi^{h}\right)_{x_{r} \bar{x}_{r}, m_{r}}\right|^{2} h_{1} \cdots h_{h}\right)^{1 / 2} .
\end{aligned}
$$

Theorem 3.1 Let $\tau$ and $|h|=\sqrt{h_{1}^{2}+\cdots+h_{n}^{2}}$ be sufficiently small positive numbers. Then, solutions of difference scheme (26) satisfy the following stability and almost coercivity estimates:

$$
\begin{aligned}
& \left\|\left\{u_{k}^{h}\right\}_{-N}^{N-1}\right\|_{C\left([-1,1]_{\tau}, L_{2 h}\right)} \leq M\left[\left\|\left\{f_{k}^{h}\right\}_{-N+1}^{-1}\right\|_{C\left([-1,0]_{\tau}, L_{2 h}\right)}\right. \\
& \left.+\left\|\left\{g_{k}^{h}\right\}_{1}^{N-1}\right\|_{C([0,1] \tau}, L_{2 h}\right) \\
& \left.\left\|\left\{\tau^{-2}\left(u_{k+1}^{h}-2 u_{k}^{h}+u_{k-1}^{h}\right)\right\}_{1}^{N-1}\right\|_{C\left([0,1]_{\tau}, L_{2 h}\right)}+\left\|\left\{u_{k}^{h}\right\}_{1}^{N-1}\right\|_{C\left([0,1]_{\tau}, W_{2 h}^{2}\right)}\right] \\
& +\left\|\left\{\tau^{-1}\left(u_{k}^{h}-u_{k-1}^{h}\right)\right\}_{-N+1}^{0}\right\|_{C\left([-1,0]_{\tau}, L_{2 h}\right)}+\left\|\left\{\frac{u_{k}^{h}+u_{k-1}^{h}}{2}\right\}_{-N+1}^{0}\right\| \|_{C\left([-1,0]_{\tau}, W_{2 h}^{2}\right)} \\
& \leq M\left[\left\|f_{0}^{h}\right\|_{L_{2 h}}+\left\|f_{-1}^{h}\right\|_{L_{2 h}}+\left\|g_{1}^{h}\right\|_{L_{2 h}}+\left\|\varphi^{h}\right\|_{W_{2 h}^{2}}+\tau\left\|f_{0}^{h}\right\|_{W_{2 h}^{1}}+\tau\left\|f_{-1}^{h}\right\|_{W_{2 h}^{1}}\right. \\
& \left.\quad+\tau\left\|g_{1}^{h}\right\|_{W_{2 h}^{1}}+\ln \frac{1}{\tau+|h|}\left[\left\|\left\{f_{k}^{h}\right\}_{-N+1}^{-1}\right\|_{C\left([-1,0]_{\tau}, L_{2 h}\right)}+\left\|\left\{g_{k}^{h}\right\}_{1}^{N-1}\right\|_{C\left([0,1]_{\tau}, L_{2 h}\right)}\right]\right],
\end{aligned}
$$


where $M$ is independent not only of $\tau, h, \varphi^{h}(x)$ but also of $f_{k}^{h},-N+1 \leq k \leq 0$ and $g_{k}^{h}(x)$, $1 \leq k \leq N-1$.

The proof of Theorem 3.1 is based on Theorem 2.1, Theorem 2.2, the symmetry properties of the difference operator $A_{h}^{x}$ defined by formula (24) in $L_{2 h}$, the estimate

$$
\min \left\{\ln \frac{1}{\tau}, 1+\left|\ln \left\|A_{h}^{x}\right\|_{L_{2 h} \rightarrow L_{2 h}}\right|\right\} \leq M \ln \frac{1}{\tau+|h|}
$$

and the following theorem in $L_{2 h}$ :

Theorem 3.2 For the solution of the elliptic difference problem

$$
A_{h}^{x} u^{h}(x)=\omega^{h}(x), \quad x \in \Omega_{h}, \quad u^{h}(x)=0, \quad x \in S_{h}
$$

the following coercivity inequality holds [23]:

$$
\sum_{r=1}^{n}\left\|\left(u^{h}\right)_{\bar{x}_{r} x_{r}, m_{r}}\right\|_{L_{2 h}} \leq M\left\|\omega^{h}\right\|_{L_{2 h}} .
$$

Here, $M$ is independent of $h$ and $\omega^{h}$.

Theorem 3.3 Let $\tau$ and $|h|$ be sufficiently small positive numbers. Then, the solutions of difference scheme (26) satisfy the following coercivity stability estimates:

$$
\begin{aligned}
&\left\|\left\{\tau^{-2}\left(u_{k+1}^{h}-2 u_{k}^{h}+u_{k-1}^{h}\right)\right\}_{1}^{N-1}\right\|_{C_{0,1}^{\alpha}\left([0,1]_{\tau}, L_{2 h}\right)}+\left\|\left\{\tau^{-1}\left(u_{k}^{h}-u_{k-1}^{h}\right)\right\}_{-N+1}^{0}\right\|\left\|_{\widetilde{C}_{0}^{\alpha}\left([-1,0]_{\tau}, L_{2 h}\right)}+\right\|\left\{u_{k}^{h}\right\}_{1}^{N-1} \|_{C_{0,1}^{\alpha}\left([0,1]_{\tau}, W_{2 h}^{2}\right)} \\
&+\left\|\left\{\frac{u_{k}^{h}+u_{k-1}^{h}}{2}\right\}_{-N+1}^{0}\right\|_{\widetilde{C}_{0}^{\alpha}\left([-1,0]_{\tau}, W_{2 h}^{2}\right)} \\
& \leq M\left[\left\|\varphi^{h}\right\|_{W_{2 h}^{2}}+\tau\left\|f_{0}^{h}\right\|_{W_{2 h}^{1}}+\tau\left\|f_{-1}^{h}\right\|_{W_{2 h}^{1}}+\tau\left\|g_{1}^{h}\right\|_{W_{2 h}^{1}}\right. \\
&\left.+\frac{1}{\alpha(1-\alpha)}\left[\left\|\left\{f_{k}^{h}\right\}_{-N+1}^{-1}\right\|_{C_{0}^{\alpha}\left([-1,0]_{\tau}, L_{2 h}\right)}+\left\|\left\{g_{k}^{h}\right\}_{1}^{N-1}\right\|_{C_{0,1}^{\alpha}\left([0,1]_{\tau}, L_{2 h}\right)}\right]\right] \\
&\left\|\left\{\tau^{-2}\left(u_{k+1}^{h}-2 u_{k}^{h}+u_{k-1}^{h}\right)\right\}_{1}^{N-1}\right\|_{C_{0,1}^{\alpha}\left([0,1]_{\tau}, L_{2 h}\right)}+\left\|\left\{\frac{u_{k}^{h}+u_{k-1}^{h}}{2}\right\}_{-N+1}^{0}\right\| \widetilde{C}_{0}^{\alpha}\left([-1,0]_{\tau}, W_{2 h}^{2}\right) \\
&+\left\|\left\{\tau^{-1}\left(u_{k}^{h}-u_{k-1}^{h}\right)\right\}_{-N+1}^{0}\right\|\left\|_{\left.\widetilde{C}_{0}^{\alpha}([-1,0]]_{\tau}, L_{2 h}\right)}+\right\|\left\{u_{k}^{h}\right\}_{1}^{N-1} \|_{C_{0,1}^{\alpha}\left([0,1]_{\tau}, W_{2 h}^{2}\right)} \\
& \leq M\left[\left\|\varphi^{h}\right\|_{W_{2 h}^{2}}+\tau\left\|f_{0}^{h}\right\|_{W_{2 h}^{1}}+\tau\left\|_{-1}^{h}\right\|_{W_{2 h}^{1}}+\tau\left\|g_{1}^{h}\right\|_{W_{2 h}^{1}}\right. \\
&\left.+\frac{1}{\alpha(1-\alpha)}\left[\left\|\left\{f_{k}^{h}\right\}_{-N+1}^{-1}\right\|_{\left.\widetilde{C}_{0}^{\alpha}([-1,0]]_{\tau}, L_{2 h}\right)}+\left\|\left\{g_{k}^{h}\right\}_{1}^{N-1}\right\|_{C_{0,1}^{\alpha}\left([0,1]_{\tau}, L_{2 h}\right)}\right]\right] .
\end{aligned}
$$

Here, $M$ is independent not only of $\tau, h, \varphi^{h}(x)$ but also of $f_{k}^{h},-N+1 \leq k \leq 0$ and $g_{k}^{h}(x)$, $1 \leq k \leq N-1$. 
Table 1 Error analysis for the solution $u(t, x)$

\begin{tabular}{lccc}
\hline Method & $\boldsymbol{N}=\boldsymbol{M = 3 0}$ & $\boldsymbol{N}=\boldsymbol{M = 6 0}$ & $\boldsymbol{N}=\boldsymbol{M = 9 0}$ \\
\hline 1st order of accuracy d. s. & 0.042169 & 0.021639 & 0.014546 \\
2nd order of accuracy d. s. & 0.000908 & 0.000227 & 0.000101 \\
\hline
\end{tabular}

The proof of Theorem 3.3 is based on the abstract Theorem 2.3, Theorem 3.2, and the symmetry properties of the difference operator $A_{h}^{x}$ defined by formula (24).

\section{Numerical Analysis}

The theoretical statements for the solution of these difference schemes are supported by the results of numerical experiments of the nonlocal boundary value problem

$$
\left\{\begin{array}{l}
\frac{\partial u}{\partial t}+\frac{\partial u}{\partial x}\left((1+x) \frac{\partial u}{\partial x}\right)=f(t, x), \\
f(t, x)=\left(-2 e^{-t}+1-t\right) \sin x+\left(e^{-t}+t\right)(\cos x-x \sin x), \\
\quad-1<t \leq 0,0<x<\pi, \\
\frac{\partial^{2} u}{\partial t^{2}}+\frac{\partial u}{\partial x}\left((1+x) \frac{\partial u}{\partial x}\right)=g(t, x), \\
g(t, x)=-t \sin x+\left(e^{-t}+t\right)(\cos x-x \sin x), \quad 0<t<1,0<x<\pi, \\
u(1, x)=\frac{1}{2} u(-1, x)+\frac{1}{2} u\left(-\frac{1}{2}, x\right)+\varphi(x), \\
\varphi(x)=\left(e^{-1}-\frac{e}{2}-\frac{1}{2} e^{\frac{1}{2}}+\frac{7}{4}\right) \sin x, \quad 0 \leq x \leq \pi, \\
u(t, 0)=u(t, \pi)=0, \quad-1 \leq t \leq 1
\end{array}\right.
$$

for the elliptic-parabolic equation. The exact solution of this problem is

$$
u(t, x)=\left(e^{-t}+t\right) \sin x
$$

For the comparison, the errors computed by the following formula

$$
E_{M}^{N}=\max _{\substack{-N \leq k \leq N \\ 1 \leq n \leq M-1}}\left|u\left(t_{k}, x_{n}\right)-u_{n}^{k}\right|
$$

are recorded for different values of $N$ and $M$, where $u\left(t_{k}, x_{n}\right)$ represents the exact solution and $u_{n}^{k}$ represents the numerical solution at $\left(t_{k}, x_{n}\right)$. The results are shown in Table 1 for $N=M=30,60$ and 90 respectively.

Therefore, the results indicate that the second order of accuracy difference scheme is more accurate than the first order of accuracy difference scheme.

Competing interests

The authors declare that they have no competing interests.

Authors' contributions

The manuscript was drafted by OG and it is based on his $\mathrm{PhD}$ thesis. AA is the supervisor of the thesis and gave detailed comments on the manuscript. All authors read and approved the final manuscript.

\section{Author details}

${ }^{1}$ Department of Mathematics, Fatih University, Büyükçekmece, Istanbul, Turkey. ${ }^{2}$ Department of Mathematics, ITTU, Ashgabat, Turkmenistan. 


\section{Acknowledgement}

The authors are very grateful to Prof. P. E. Sobolevskii (Jerusalem, Israel) for valuable comments to the improvement of this article.

Received: 13 March 2012 Accepted: 10 July 2012 Published: 27 July 2012

\section{References}

1. Salakhitdinov, MS: Equations of Mixed-Composite Type. FAN, Tashkent (1974)

2. Chipot, M, Lovat, B: Existence and uniqueness results for a class of nonlocal elliptic and parabolic problems. Dyn. Contin. Discrete Impuls. Syst. 8(1), 35-51 (2001)

3. Gulin, AV, Ionkin, NI, Morozova, VA: Stability of a nonlocal two-dimensional finite-difference problem. Differ. Equ 37(7), 970-978 (2001)

4. Agarwal, RP, Bohner, M, Shakhmurov, VB: Maximal regular boundary value problems in Banach-valued weighted space. Bound. Value Probl. 1, 9-42 (2005)

5. Gordeziani, DG, Avalishvili, GA: Time-nonlocal problems for Schrödinger-type equations: I. Problems in abstract spaces. Differ. Equ. 41(5), 703-711 (2005)

6. Gordeziani, N, Natalini, P, Ricci, PE: Finite-difference methods for solution of nonlocal boundary value problems. Comput. Math. Appl. 50, 1333-1344 (2005)

7. Berdyshev, AS, Karimov, ET: Some nonlocal problems for the parabolic-hyperbolic type equation with non-characteristic line of changing type. Cent. Eur. J. Math. 4(2), 183-193 (2006)

8. Martín-Vaquero, J, Vigo-Aguiar, J: On the numerical solution of the heat conduction equations subject to nonlocal conditions. Appl. Numer. Math. 59, 2507-2514 (2009)

9. Ashyralyev, A, Gercek, O: Finite difference method for multipoint nonlocal elliptic-parabolic problems. Comput. Math. Appl. 2010(7), 2043-2052 (2010)

10. Gordeziani, DG, Berikelashvili, GK: On a nonlocal generalization of the biharmonic Dirichlet problem. Differ. Equ. 46(3), 321-328 (2010)

11. Gavrilyuk, IP, Makarov, VL, Sytnyk, DO, Vasylyk, VB: Exponentially convergent method for the $m$-point nonlocal problem for a first order differential equation in Banach space. Numer. Funct. Anal. Optim. 31(1), 1-21 (2011)

12. Jangveladze, TA, Lobjanidze, GB: On a nonlocal boundary value problem for a fourth-order ordinary differential equation. Differ. Equ. 47(2), 335-343 (2011)

13. Martín-Vaquero, J, Queiruga-Dios, A, Encinas, AH: Numerical algorithms for diffusion-reaction problems with non-classical conditions. Appl. Math. Comput. 218(9), 5487-5495 (2012)

14. Vragov, VN: Boundary Value Problems for Nonclassical Equations of Mathematical Physics. Textbook for Universities. NSU Publishing house, Novosibirsk (1983)

15. Kröner, D, Rodriques, JF: Global behaviour for bounded solutions of a porous media equation of elliptic-parabolic type. J. Math. Pures Appl. 64(2), 105-120 (1985)

16. Nakhushev, AM: Equations of Mathematical Biology. Textbook for Universities. Vysshaya Shkola, Moskow (1995)

17. Ashyralyev, A, Soltanov, H: On one difference schemes for an abstract nonlocal problem generated by the investigation of the motion of gas on the homogeneous space. In: Modeling Processes of Exploitation of Gas Places and Applied Problems of Theoretical Gasohydrodynamics, pp. 147-154. Ashgabat (1998)

18. Diaz, JI, Lerena, MB, Padial, JF, Rakotoson, JM: An elliptic-parabolic equation with a nonlocal term for the transient regime of a plasma in a stellarator. J. Differ. Equ. 198(2), 321-355 (2004)

19. Gercek, O: Difference schemes of nonlocal boundary value problems for elliptic-parabolic differential equations. PhD thesis, Yildiz Teknik University (2010)

20. Ashyralyev, A, Gercek, O: On multipoint nonlocal elliptic-parabolic difference problems. Vestn. Odessa Nat. Univ. Math. Mech. 15(19), 135-156 (2010)

21. Sobolevskii, PE: The coercive solvability of difference equations. Dokl. Akad. Nauk SSSR 201(5), 1063-1066 (1971)

22. Sobolevskii, PE: On the stability and convergence of the Crank-Nicolson scheme. In: Variational-Difference Methods in Mathematical Physics, pp. 146-151. Vychisl. Tsentr Sibirsk. Otdel. Akad. Nauk SSSR, Novosibirsk (1974)

23. Sobolevskii, PE: Difference Methods for the Approximate Solution of Differential Equations. Voronezh State University Press, Voronezh (1975)

24. Ashyralyev, A, Sobolevskii, PE: Coercive stability of a Crank-Nicolson difference scheme in $\widetilde{C}_{0}^{\alpha}$ spaces. In: Approximate Methods for Investigations of Differential Equations and Their Applications, pp. 16-24. Kuybishev (1982)

doi:10.1186/1687-2770-2012-80

Cite this article as: Gercek and Ashyralyev: On a difference scheme of the second order of accuracy for

elliptic-parabolic equations. Boundary Value Problems 2012 2012:80. 